Revista de BIOLOGía TROPICAL

\title{
¿Qué factores determinan la distribución altitudinal de los peces de ríos tropicales andinos?
}

\author{
Evans De La Barra ${ }^{1,2,4 *}$, José Zubieta ${ }^{1,2}$, Gastón Aguilera ${ }^{3}$, Mabel Maldonado ${ }^{1}$, Marc Pouilly² \& \\ Thierry Oberdorff ${ }^{2}$ \\ 1. Unidad de Limnología y Recursos Acuáticos (ULRA), Universidad Mayor de San Simón (UMSS), Sucre y Parque \\ La Torre s/n, Cochabamba, Estado Plurinacional de Bolivia; cyprinodon@gmail.com, pp_zubiet@yahoo.com, \\ mabelmaldonado.m@fcyt.umss.edu.bo \\ 2. UMR 'BOREA', IRD 207/CNRS 7208/MNHN/UPCM, UNICAEN, Muséum National d'Histoire Naturelle, 43 rue \\ Cuvier, 75231 Paris Cedex, FRANCIA; pouilly@ird.fr, thierry.oberdorff@ird.fr \\ 3. Fundación Miguel Lillo, UEL-CONICET, Miguel Lillo 251, Tucumán (CP4000), Argentina; gaguilera@lillo.org.ar \\ 4. Universidad Nacional de Tucumán, Miguel Lillo 251, Tucumán (CP4000), Argentina.
}

$$
\text { Recibido 26-III-2015. Corregido 07-IX-2015. Aceptado 05-X-2015. }
$$

\begin{abstract}
Which factors determine the altitudinal distribution of tropical Andean riverine fishes? Altitudinal gradients represent an appropriate system to assess whether there is a relationship between richness patterns, environmental variables, and the ecological processes that determine the species type and number inhabiting a given area. In mountain streams freshwater fishes, the most prevalent relationship is a monotonic decrease in species richness with elevation. The objective of this study was to evaluate four hypotheses that can explain the negative relationship between local fish species richness and altitude, 1) the hypothesis of decreasing energy availability, 2) the hypothesis of increasing climate severity, 3) the hypothesis of habitat diversity, and 4) the hypothesis of isolation by physical severity of the environment. Fish and macro-invertebrates were collected following standard methods from 83 sites (between 200-4 000 meters) of two river basins in the Bolivian Amazon. The first hypothesis was tested by analyzing relationships between the density of macro-invertebrates, the richness of invertivorous fish species and altitude; while the second and third hypotheses were assessed by a multiple regression analysis (GLM) between fish species richness and several local and regional factors. Besides, assemblage dissimilarity between sites along the altitudinal gradient was analyzed using $\beta$ sim and $\beta$ ness indices. Fish richness decreases linearly with increasing altitude. The density of macro-invertebrates tends to increase at higher altitudes, contrary to invertivorous fish species richness, suggesting that energy availability is not a limiting factor for fish species colonization. The GLM explained $86 \%$ of the variation in fish species richness, with a significant contribution of water temperature, maximum slope in the river mainstem, and stream width. There is a higher species turnover ( $\beta$ sim) between sites at low elevation. Inversely, $\beta$ ness shows higher values in the upper parts, corresponding to change in assemblages mainly due to species loss. Taken together, these results suggest that climatic and physical severities create strong barriers to colonization, further explaining the decrease in fish richness along the altitudinal gradient. Rev. Biol. Trop. 64 (1): 157-176. Epub 2016 March 01.
\end{abstract}

Key words: species richness, Bolivian Amazon, mountain streams, maximum slopes in the main river.

Los procesos y factores ecológicos involucrados en la distribución de las especies a lo largo del gradiente altitudinal no han sido totalmente esclarecidos y varían según los organismos y ecosistemas estudiados (Lomolino, 2001). Dos patrones generales que representan la relación entre la riqueza de especies y la altura han sido descritos (Nogués-Bravo,
Araujo, Romdal, \& Rahbek, 2008; Rahbek, 1995): El primero de ellos es una relación unimodal, caracterizada por una mayor riqueza en alturas intermedias, y una menor riqueza en las partes alta y baja. El segundo patrón es una relación lineal inversa entre la riqueza y la altura. Para los peces, este último patrón parece prevalecer y ocurre tanto en la región tropical 
(Jaramillo-Villa, Maldonado-Ocampo, \& Escobar, 2010; Lujan et al., 2013; Pouilly, Barrera, \& Rosales, 2006) como en zonas templadas (Rahel, 1991). Se han propuesto varias hipótesis que intentan explicar la disminución gradual de la riqueza de especies con el incremento de la altura, mismas que pueden resumirse en tres: 1) la disminución de la energía disponible (Wright, 1983), 2) el aumento del rigor climático o hipótesis de los límites fisiológicos (Turner, Gatehouse, \& Corey, 1987), y 3) la disminución del tamaño y de la diversidad de hábitats (Williams, 1964). La primera hipótesis relaciona el aumento de la altura con una disminución de los recursos disponibles debido a la reducción de la productividad primaria y/o de la diversidad de recursos. La segunda hipótesis relaciona el aumento de la altura con el rigor del clima, el cual está asociado con el enfriamiento adiabático a mayor altura (Rahbek, 1995). La tercera hipótesis relaciona el aumento de la altura con una disminución del tamaño y de la diversidad de hábitats disponibles para los peces. En este trabajo evaluamos estas tres hipótesis e incorporamos una cuarta hipótesis basada en 4) la severidad de las condiciones físicas del hábitat a través de un gradiente de altitud. Esta cuarta hipótesis, considerando que las vías de dispersión de las especies de peces se hacen preferentemente de abajo hacia arriba, en relación con la altura a la dificultad de colonización, consecuencia del aumento de la severidad de las condiciones físicas, tales como variaciones abruptas de las pendientes del río que crean barreras para la dispersión.

Probamos estas cuatro hipótesis comparando las comunidades de peces a lo largo del gradiente altitudinal en dos cuencas de los Andes amazónicos de Bolivia. Estas dos cuencas se encuentran geográficamente próximas entre sí, los ríos son similares en sus condiciones climáticas, tipo de hábitats y condiciones edáficas. Sin embargo, las pendientes de los ríos varían de manera significativa. Esta configuración nos permite responder a tres preguntas: (1) ¿Cómo varía la riqueza y estructura de las comunidades de peces a lo largo del gradiente altitudinal?, (2) ¿Qué grado de similitud existe entre los patrones de distribución de las comunidades de peces de las dos cuencas?, (3) ¿Qué se puede deducir de esta comparación sobre los factores que afectan la distribución de las especies a lo largo del gradiente de altura?

\section{MATERIALES Y MÉTODOS}

Sitios y área de estudio: El estudio se realizó en la vertiente oriental de la cordillera de los Andes, en las cuencas de los ríos Espíritu Santo y Alto Beni, ambos afluentes del río Madera-Amazonas, ubicados entre $15^{\circ} 00^{\prime} 00^{\prime \prime}$ $18^{\circ} 00^{\prime} 00^{\prime}$ S y $65^{\circ} 00^{\prime} 00^{\prime \prime}-68^{\circ} 00^{\prime} 00^{\prime \prime}$ W (Fig. 1). La zona se caracteriza por tener una fuerte estacionalidad climática con las mayores precipitaciones entre enero y marzo, y un periodo de estiaje entre mayo y noviembre. Debido al amplio rango altitudinal, se presentan varios bioclimas desde pluvio-estacional húmedo hasta pluvial híper-húmedo, con altas variaciones de temperatura, frío en las partes altas de las montañas a calientes en las zonas bajas (Navarro \& Maldonado, 2002). Los ríos y arroyos en esta zona forman valles angostos con sustrato grueso y altas velocidades. La cuenca del río Espíritu Santo presenta un área menor y pendientes más abruptas $\left(2354 \mathrm{~km}^{2}\right.$ y $8 \%$ respectivamente) que la del Alto Beni (11761 km² y $1 \%)$.

Se seleccionaron 83 sitios de muestreo en arroyos de menos de 10 metros de ancho con baja perturbación antropogénica, 54 sitios en la cuenca del río Alto Beni (300 a 2000 m) y 29 sitios en la cuenca del río Espíritu Santo (200 a $4000 \mathrm{~m}$ ).

Datos biológicos: Se realizaron muestreos de peces en la época seca de los años 2008 y 2009 (Agosto-Octubre). En cada sitio se recolectó en un tramo de 20 a 30 metros de longitud del arroyo, previamente cercado en ambos extremos con redes de nylon de 2.5 mm de luz de malla (Tedesco et al., 2007). Se realizaron dos repeticiones, de pesca eléctrica a contracorriente usando un equipo marca EFKO (modelo FEG 8000) aplicando un esfuerzo constante. El método de dos barridos con pesca 


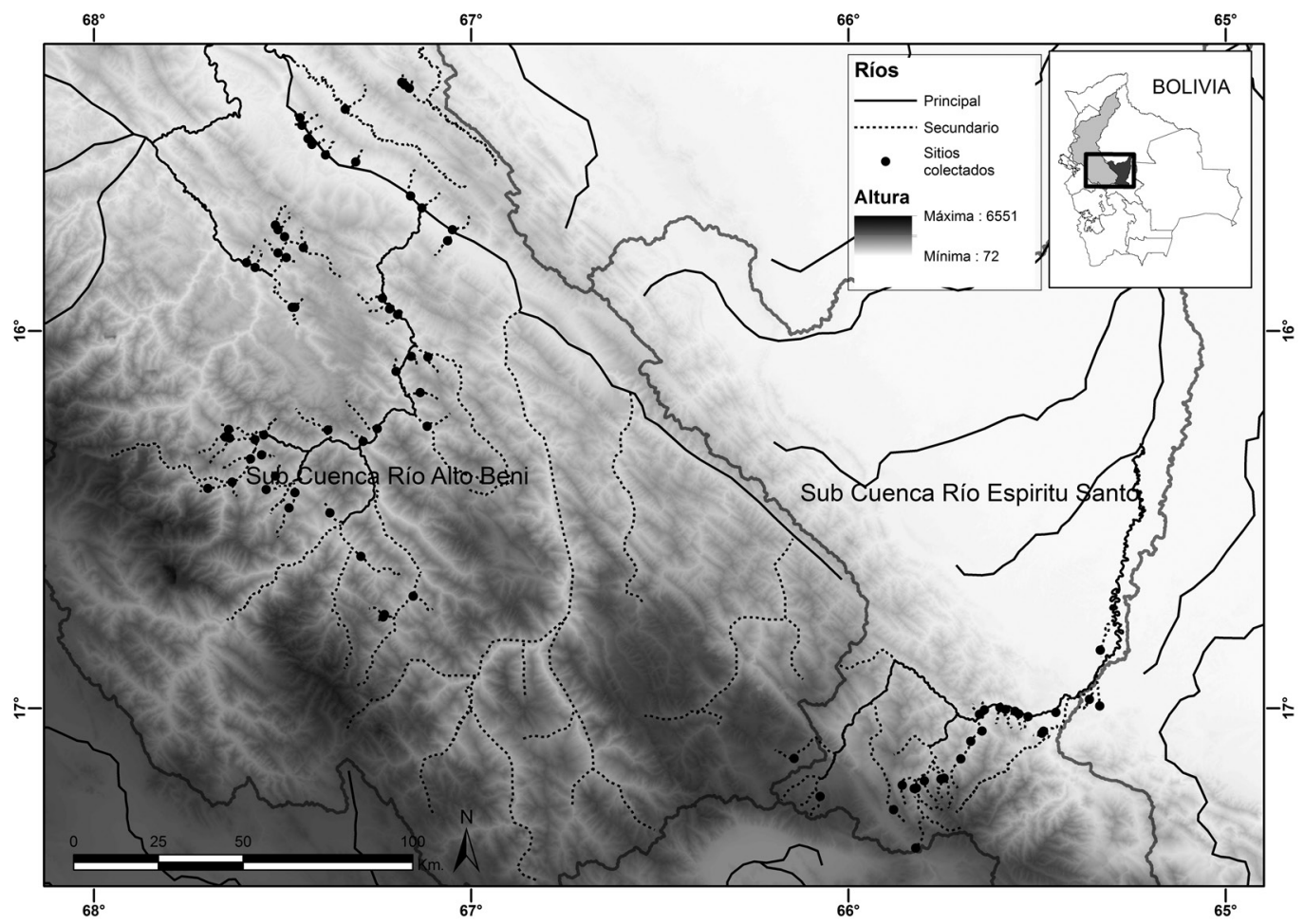

Fig. 1. Red hidrográfica de las cuencas altas del Alto Beni y del Espíritu Santo en la vertiente Amazónica de los Andes de Bolivia. $(\bullet)$ puntos de recolecta.

Fig. 1. The two Andean drainage basins analyzed (i.e. upper Beni and Espíritu Santo drainages) (•) sampling sites.

eléctrica proporcionó estimaciones confiables de abundancia y riqueza. El $62 \%$ ( \pm 11 DS) de la abundancia total de peces y el $90 \%$ ( \pm 12 DS) de las especies fueron capturadas en la primera pasada, lo que demostró la efíciencia de la metodología aplicada (Cattaneo, Hugueny, \& Lamouroux, 2003). Los peces antes de ser formolizados fueron anestesiados en una solución de eugenol (10 gotas/L de $\left.\mathrm{H}_{2} \mathrm{O}\right)$ durante 10 minutos.

En el laboratorio, la identificación taxonómica fue realizada a nivel de especie, y de género en algunos casos en los que no se pudo identificar la especie. Se utilizó para la identificación las claves de Eigenmann (1918), Géry (1977) y Pearson (1924). Los peces fueron depositados en el Museo de Historia Natural Alcide d'Orbigny (Cochabamba, Bolivia), y los macro-invertebrados se depositaron en la colección de la Unidad de Limnología y Recursos Acuáticos de la Universidad Mayor de San Simón (Cochabamba, Bolivia).

En cada sitio también se recolectaron macro-invertebrados bénticos usando una red Surber de $250 \mu \mathrm{m}$ de abertura de malla y 900 $\mathrm{cm}^{2}$ de área recolectora (Moya et al,. 2011). Se tomó una muestra compuesta de cinco réplicas representativas en las zonas de rabión de cada estación. Los organismos fueron contados e identificados a nivel taxonómico de familia, excepto para los grupos de Oligochaeta, Mollusca y Crustacea, que fueron identificados a niveles superiores. Se calculó la riqueza de especies y la densidad de individuos (número de individuos $/ \mathrm{m}^{2}$ ) para cada sitio. Estas dos variables fueron utilizadas para evaluar la hipótesis de que la energía disponible disminuye a medida que aumenta la altura, sabiendo que los 
macro-invertebrados acuáticos constituyen el alimento principal de la mayoría de las especies de peces en la zona muestreada.

Variables ambientales consideradas: $\mathrm{Se}$ registraron los valores de dos tipos de variables: variables locales (profundidad y temperatura del agua, ancho del río, distancia a la fuente de colonización y altura) y variables regionales (pendiente máxima en el río principal y la cuenca como variable categórica) (Apéndice 1.). La profundidad media del agua en cada estación de muestreo fue calculada con base en medidas efectuadas con una varilla graduada en intervalos de $1 \mathrm{~m}$ en cada uno de varios transectos perpendiculares a la dirección del río medidos cada $3 \mathrm{~m}$. Estos transectos perpendiculares sirvieron igualmente para calcular la media del ancho de río. Ya que las medidas de temperatura tomadas en campo no se realizaron de forma estandarizada (a la misma hora del día), los datos de temperatura fueron generados con base en una regresión lineal $(\mathrm{y}=$ $0.000001 \mathrm{x}^{2}-0.0095 \mathrm{x}+26.85$ donde $\mathrm{x}=$ altura, $\mathrm{y}=$ temperatura) desarrollada por Lujan et al. (2013) en ambientes acuáticos similares a los de este estudio. Esta relación se pudo validar con datos de 54 sitios en la cuenca del río Alto Beni, donde los valores observados y estimados de temperatura se encontraron significativamente correlacionados $(\mathrm{r}=0.805, \mathrm{p}<0.001)$. La distancia a la fuente de colonización para cada sitio de muestreo se estimó como la distancia de río entre el sitio y el río principal donde confluyen todos los arroyos en cada una de las dos cuencas. Estas distancias se midieron con el programa ArcGis v10.1. Las pendientes mayores en el río principal fueron incorporadas en el análisis para evaluar la influencia de las barreras de dispersión que los peces tienen que superar para poder colonizar las zonas altas de la cuenca. Los datos de distancia y diferencias de altura fueron tomados del programa Google Earth (DEM SRTM30). La pendiente fue estimada cada $100 \mathrm{~m}(\mathrm{PMRP}=(\Delta$ Altura $) /(100$ m)) a lo largo del río principal al cual drenan los arroyos muestreados. El valor asignado a cada sitio fue el valor máximo de pendiente entre todas las secciones medidas en el río principal, entre su confluencia con el arroyo en el cual se pescó y la confluencia del mismo río principal con el arroyo más próximo río abajo donde también se pescó (Apéndice 2).

Antes efectuar los análisis estadísticos, las variables fueron transformadas para mejorar su normalidad cuando fue necesario, la riqueza de peces, densidad de macro invertebrados, la temperatura, el ancho del río, la distancia a la fuente, la profundidad y la altura fueron normalizadas en $\log _{10}$, la variable pendiente máxima en el río principal fue normalizada usando el arco seno de la raíz cuadrada del valor. Para analizar el tipo de relación (lineal o unimodal) entre la altura y la riqueza de peces se realizó un análisis de regresión múltiple entre la riqueza, la altura y su valor al cuadrado. Para determinar si la riqueza de los ríos Espíritu Santo y Alto Beni responde de manera similar en función a la altura se ha realizado un análisis de regresión múltiple entre la riqueza de peces, la altura, la cuenca y la interacción entre la cuenca y la altura. Un efecto significativo de la cuenca, significa que el intercepto de las relaciones entre riqueza y altura es diferente entre cuencas. Un efecto significativo de la interacción entre la cuenca y la altura, significa que las pendientes de las relaciones son diferentes entre las dos cuencas.

Para evaluar la hipótesis de la disminución de la energía disponible (alimento) con el aumento de la altura (primera hipótesis), se examinó la relación entre la densidad de macro-invertebrados acuáticos, alimento principal de la mayoría de las especies de peces en la zona muestreada (29 de 55 especies; Ibañez et al., 2007; Pouilly et al., 2006; Tedesco et al., 2007) y el gradiente altitudinal. Paralelamente, se examinó la relación entre el gradiente altitudinal y su cuadrado frente a la riqueza de las 29 especies invertívoras.

Para evaluar las hipótesis de rigor climático (segunda hipótesis), de disminución del tamaño del hábitat (tercera hipótesis) y de severidad en las condiciones físicas (cuarta hipótesis), se realizó un análisis de regresión múltiple (General Lineals Models) en el 
programa $\mathrm{R}$, entre la riqueza y las distintas variables ambientales relacionadas a cada hipótesis a evaluar. Para la hipótesis de rigor climático, se usó la variable "temperatura del agua", que debería actuar como un filtro fisiológico sobre los organismos. Dentro de la hipótesis de severidad en las condiciones físicas se evaluó el efecto de la variable "pendientes máximas en el río principal" como dificultad de dispersión. Las variables "ancho de río", "profundidad del agua" y "distancia a la fuente de colonización" se utilizaron para evaluar el efecto de la disponibilidad y diversidad de hábitats. Las variables "ancho de río" y "profundidad del agua" son variables que evalúan la disponibilidad y diversidad al nivel local (del sitio). La variable "distancia a la fuente de colonización" (o su opuesto "distancia a la fuente") evalúa de manera indirecta la disponibilidad y diversidad de hábitats a lo largo del gradiente longitudinal a través del aumento del volumen de agua disponible para los peces (Petts \& Calow, 1996). Se añadió la variable categórica "cuenca" a este análisis para evaluar si la diferencia de riqueza entre las cuencas de los ríos Espíritu Santo y Alto Beni se debe solo a un efecto de las variables analizadas.

Para determinar posibles efectos cuadráticos, se incluyeron en el análisis los cuadrados de cada una de las variables analizadas. Como existía co-linealidad entre algunas variables de respuesta (mediana de los coeficientes de correlación entre variables $=0.2$; máxima $=$ 0.9) se utilizó el modelo de regresión múltiple completo que es más adecuado que la selección "stepwise" para este tipo de configuración (Whittingham, Stephens, Bradbury, \& Freckleton, 2006). Sin embargo, para reducir el número de variables en el modelo, hemos utilizado la selección stepwise opción backward.

Se determinó el intervalo de distribución altitudinal de las especies de peces, tomando como límites los sitios con alturas extremas donde la especie fue encontrada. Para constatar la existencia de una colonización constante de las especies desde las partes de menor altura hacia las partes altas de las cuencas, y desvirtuar la posible residencia de especies en diferentes niveles de altura, realizamos un análisis de correlación entre la densidad de cada una de las especies de peces y la altura, donde la mayor densidad debería corresponder a los lugares donde las especies tienen sus fuentes de colonizadores (Jaramillo-Villa et al. 2010).

La hipótesis de severidad de las condiciones físicas del hábitat que se presentan río arriba fue adicionalmente evaluada con base en los índices de disimilitud de Simpson ( $\beta$ sim) y de Nestedness ( $\beta$ nes) que cuantifican los procesos de colonización y desaparición de las especies. En estos análisis, los sitios de recolecta se agruparon por intervalos altitudinales de 200 m. En cada intervalo de altitud se calculó el índice de disimilitud de Sorensen o disimilitud total de la diversidad beta: $\beta$ sor $=(b+c) /(2 a$ $+b+c)$. Donde " $a$ " es el número de especies comunes en ambos sitios, "b" es el número de especies que ocurren en el primer sitio pero no en el segundo y "c" es el número de especies que ocurren en el segundo sitio pero no en el primero. De este modo se obtiene una matriz de disimilitud con un valor por cada par de sitios comparados.

El $\beta$ sor se descompuso en sus dos componentes (Baselga, 2010): 1) el índice de disimilitud de Simpson $(\beta \operatorname{sim}=\min (\mathrm{b}, \mathrm{c}) /(\mathrm{a}$ $+\min (\mathrm{b}, \mathrm{c})))$, y 2) el índice de disimilitud de Nestedness "anidamiento" ( $\beta$ nes), estimado por la diferencia entre el $\beta$ sor y el $\beta$ sim). El $\beta$ sim mide la tasa de reemplazo de las especies entre los sitios. El $\beta$ nes considera el grado de anidamiento de las comunidades entre sitios. Para observar la evolución de estos índices a lo largo del gradiente altitudinal, se graficaron para cada cuenca los valores por rango de altitud en porcentaje de la disimilitud total ( $\beta$ sor).

\section{RESULTADOS}

En el estudio se registraron 55 especies de peces, 38 especies en la cuenca del río Alto Beni y 41 en la cuenca del río Espíritu Santo (Apéndice 3).

Relación riqueza de especies de pecesvariables ambientales: La riqueza de especies 
en ambas cuencas disminuye a medida que se incrementa la altura (Fig. 2). El modelo de regresión múltiple (Cuadro 1) de la riqueza en función de la altura, la cuenca y la interacción cuenca-altura, mostró que el intercepto de las relaciones es diferente entre cuencas (efecto de la cuenca significativo; $\mathrm{P}=0.02$ ), indicando que existen diferencias significativas de riqueza entre los arroyos de las dos cuencas. Sin embargo, el modelo no muestra una interacción significativa cuenca-altura $(\mathrm{P}=0.09)$, lo cual demuestra que las pendientes de las relaciones entre la riqueza y la altura no son estadísticamente diferentes entre las dos cuencas, y sugiere por lo tanto, que los procesos ecológicos que actúan son comparables (Cuadro 1).

Usando los datos de ambas cuencas en conjunto, evaluamos el tipo de relación que existe entre la riqueza y las principales variables ambientales seleccionadas por el modelo de regresión múltiple (Cuadro 2) (i.e. pendiente máxima, ancho del rio y temperatura). Los resultados indican que, al igual que con la altura, existe una relación negativa entre la

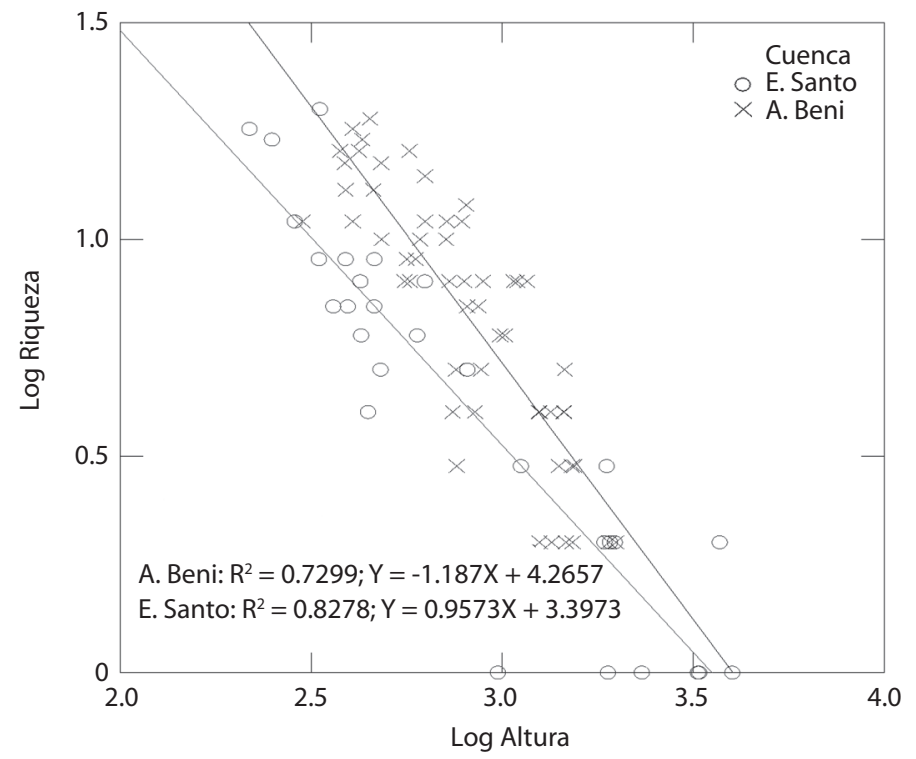

Fig. 2. Relación entre la riqueza de especie de peces y la altura para las 2 cuencas analizadas.

Fig. 2. Relationships between local fish species richness and altitude for the two drainage basins analyzed.

CUADRO 1

Modelo de regresión múltiple que analiza la variación de la riqueza local de especies de peces como una función de la altura, la cuenca y la interacción entre cuenca-altura

TABLE 1

Multiple regression model analyzing the variation in local fish species richness as a function of altitude, drainage and the interaction between drainage and altitude

\begin{tabular}{lccccc}
\multicolumn{1}{c}{ Efecto } & Coeficiente & Error estándar & Coeficiente estándar & Tolerancia & P \\
Constante & 3.397 & 0.226 & 0 & - & $<0.000$ \\
Cuenca & 0.868 & 0.381 & 1.176 & 0.01 & 0.025 \\
$\log _{10}$ (Altura) & -0.957 & 0.077 & -0.776 & 0.652 & $<0.000$ \\
$\log _{10}$ (Altura)*Cuenca & -0.226 & 0.13 & -0.897 & 0.009 & 0.086 \\
\hline
\end{tabular}


CUADRO 2

Análisis de regresión lineal múltiple de la riqueza en función a las variables ambientales (Modelo Completo)

TABLE 2

Multiple regression model analyzing the variation in local fish species richness as a function of the environmental variables (full model)

\begin{tabular}{|c|c|c|c|c|c|c|}
\hline Efecto & Coeficiente & Error estándar & Coeficiente estándar & Tolerancia & $P$ & Significancia \\
\hline CONSTANTE & 1.904 & 1.321 & 0 & - & 0.154 & \\
\hline Cuenca & 0.237 & 0.079 & 0.321 & 0.177 & 0.004 & $* *$ \\
\hline $\log _{10}($ temperatura $)$ & -5.738 & 2.443 & -2.348 & 0.002 & 0.022 & $* *$ \\
\hline $\log _{10}(\text { temperatura })^{2}$ & 3.251 & 1.422 & 2.959 & 0.001 & 0.025 & $*$ \\
\hline Asen_raiz (pendiente) & -1.832 & 0.896 & -0.501 & 0.033 & 0.045 & * \\
\hline Asen_raiz (pendiente) $)^{2}$ & 5.104 & 2.233 & 0.542 & 0.036 & 0.025 & * \\
\hline $\log _{10}$ (ancho) & 0.846 & 0.398 & 0.508 & 0.035 & 0.037 & $*$ \\
\hline $\log _{10}(\text { ancho })^{2}$ & -0.44 & 0.319 & -0.336 & 0.034 & 0.172 & ns \\
\hline $\log _{10}($ distancia a la fuente $)$ & -0.296 & 0.162 & -0.279 & 0.085 & 0.073 & ns \\
\hline $\log _{10}(\text { distancia a la fuente })^{2}$ & 0.191 & 0.099 & 0.293 & 0.086 & 0.058 & ns \\
\hline $\log _{10}($ profundidad $)$ & 1.782 & 1.224 & 0.872 & 0.006 & 0.15 & $\mathrm{~ns}$ \\
\hline $\log 10{\text { (profundidad })^{2}}^{2}$ & -0.721 & 0.474 & -0.922 & 0.005 & 0.133 & ns \\
\hline $\log _{10}($ altura $)$ & -0.172 & 0.457 & -0.14 & 0.015 & 0.707 & $\mathrm{~ns}$ \\
\hline
\end{tabular}

$* * \mathrm{p}<0.02, * \mathrm{p}<0.05, \mathrm{~ns} \mathrm{p}>0.05$.

variable "pendientes máximas en el río principal" y la riqueza, aunque en valores bajos de estas pendientes se pueden observar arroyos con alta y baja riqueza. Sin embargo, a medida que se incrementa la pendiente máxima disminuyen estos contrastes de riqueza entre arroyos, tornándose homogéneo y con menor riqueza (Apéndice 4A). Por el contrario, existe una relación positiva entre la riqueza de especies y la temperatura (Apéndice 4B). De modo similar, existe una relación positiva no muy contundente entre el ancho del río y la riqueza (Apéndice 4C). Si bien estas relaciones solamente nos dan una idea de las tendencias y no explican la relación altura-riqueza, nos permiten comprender cómo cada variable ambiental se relaciona con la riqueza.

Hipótesis (1) de la energía disponible: Los resultados de la regresión entre la altura y la densidad de invertebrados muestran que existe una tendencia positiva no muy contundente entre la densidad de macro-invertebrados acuáticos y la altura $(\mathrm{P}=0.055, \mathrm{r}=0.263)$ (Apéndice 5A). La relación entre la riqueza de peces invertívoros y la altura (más su cuadrado) muestran una disminución del número de especies invertívoras con la altura $(\mathrm{p}<0.001$, $\mathrm{R}^{2}=0.562$ ) (Apéndice 5B). Estas relaciones sugieren que la cantidad de alimento no es un factor limitante para la colonización de los peces invertívoros que van desapareciendo en los ríos a mayor altura.

Hipótesis (2) de rigor climático, (3) de la diversidad de hábitats y (4) de la severidad de las condiciones físicas: El modelo de regresión múltiple completo explicó el $86 \%$ (P $<0.001)$ de la variación de la riqueza. Cuatro variables resultaron significativamente relacionadas con los cambios de riqueza de peces: "ancho del río", "temperatura del agua", "pendiente máxima en el río principal" y "cuenca" (Cuadro 2). El modelo "stepwise" confirma estos resultados y selecciona como significativas las mismas variables explicando el $84 \%$ de la variación de la riqueza (Cuadro 3 ).

Considerando los resultados del modelo "stepwise" la temperatura del agua como medida del aumento del rigor climático presenta, 
CUADRO 3

Análisis de regresión lineal múltiple de la riqueza en función a las variables ambientales (Modelo Stepwise opción Backward)

TABLE 3

Stepwise regression model (option backward) analyzing the variation in local fish species richness as a function of the environmental variables

\begin{tabular}{|c|c|c|c|c|c|}
\hline Efecto & Coeficiente & Error estándar & Coeficiente estándar & Tolerancia & $\mathrm{P}$ \\
\hline CONSTANTE & 3.153 & 0.665 & 0 & . & $<0.001$ \\
\hline Cuenca & 0.216 & 0.061 & 0.293 & 0.307 & 0.001 \\
\hline $\log _{10}$ (ancho) & 0.329 & 0.083 & 0.198 & 0.845 & $<0.001$ \\
\hline $\operatorname{LOg}_{10}$ (temperatura) & -7.214 & 1.292 & -2.952 & 0.007 & $<0.001$ \\
\hline $\log _{10}(\text { temperatura })^{2}$ & 4.041 & 0.593 & 3.678 & 0.007 & $<0.001$ \\
\hline Asen_raiz (pendiente) & -1.941 & 0.786 & -0.531 & 0.045 & 0.016 \\
\hline Asen_raiz (pendiente) $)^{2}$ & 4.678 & 2.067 & 0.497 & 0.044 & 0.026 \\
\hline
\end{tabular}

junto a su cuadrado, el mayor aporte en el análisis (66 \% de la variación). En el análisis de regresión múltiple también se evaluó la dificultad de colonización río arriba por medio de la variable 'pendientes máximas en el río principal' que, junto a su cuadrado, explicaron una parte significativa $(10 \%)$ de la variación de riqueza en el gradiente altitudinal. Esto sugiere que los aumentos de pendiente a nivel de los cauces de mayor tamaño (río abajo) generan una dificultad importante para la colonización de las especies en los ríos de mayor altura, actuando como una barrera que filtra el paso de las especies más capacitadas para superar y sobrevivir en condiciones hidráulicas más severas (Apéndice 6).

La variable 'ancho de río' explicó $13 \%$ de la variación total de la riqueza. La variable 'cuenca' representa $11 \%$ de la variación, indicando aún diferencias de riqueza entre las dos cuencas que no fueron tomadas en cuenta a través de las otras variables explicativas del modelo. Manteniéndose todo lo demás constante, los sitios de la cuenca del río Alto Beni son, en promedio, más ricos que los sitios de la cuenca del río Espíritu Santo (Fig. 2 y Cuadro 2). Por otro lado, la altura no entra como variable significativa en el modelo (Cuadro 3 ), lo cual demuestra que los factores tomados en cuenta y ligados a esta variable sintética son suficientes para explicar los cambios de riqueza en el gradiente altitudinal.
Distribución de las especies de peces a lo largo del gradiente altitudinal: Nuestros resultados muestran que tanto en la cuenca del río Alto Beni como en la del río Espíritu Santo, existe una riqueza mayor en la parte baja del gradiente. Así mismo, se muestra que las especies que están presentes en la parte alta se encuentran distribuidas en todo el gradiente, mientras que las especies que viven en alturas bajas presentan un menor rango de distribución (Fig. 3). La alta concentración de especies en la parte baja del gradiente altitudinal sugiere que la colonización de las especies se produce desde zonas de menor altura a las de mayor altura. Para evaluar esta hipótesis realizamos un análisis de correlación entre la densidad de cada una de las especies y la altura del sitio de recolecta. Los resultados muestran que 51 de las 55 especies presentan mayores densidades en las partes bajas (correlaciones negativas Apéndice 7).

Disimilitud de las comunidades de peces: Los cambios graduales de riqueza que existen entre los arroyos de la parte baja y alta del gradiente se evaluaron por medio de la descomposición del índice de disimilitud ßsor en dos subcomponentes: el $\beta$ sim que muestra la tasa de recambio de especies entre sitios y el $\beta$ nes que considera el grado de anidamiento de las comunidades. Los resultados muestran que en las partes bajas donde la temperatura 


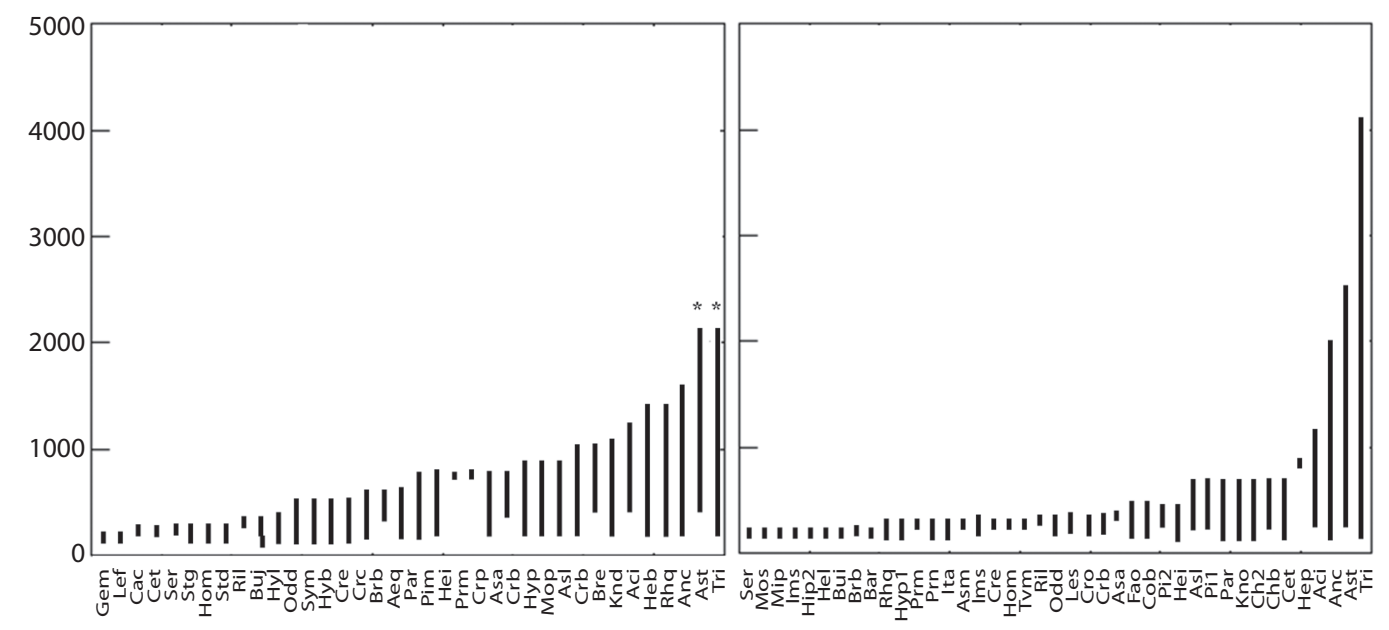

Códigos de nombres de especies

Fig. 3. Ámbito de distribución altitudinal (m) de las principales especies de peces de las cuencas del río Alto Beni (Izquierda) y cuenca del río Espíritu Santo (Derecha); ${ }^{*}=$ la parte alta del ámbito de distribución altitudinal de esas especies no fue determinado.

Fig. 3. Altitudinal range of the main fish species samples in the upper Beni (left) and the Espíritu Santo (right) drainages; $*$ = upper value of the range not determined.

del agua es mayor y las pendientes en el río principal son bajas, el $\beta$ sim es mayor y por consiguiente el $\beta$ nes menor. Esto sugiere que entre los sitios de las partes bajas los cambios de riqueza se deben a un constante recambio de especies, es decir, pérdidas y recolonizaciones continuas, o a una composición diferente en función a factores locales como el tamaño y variabilidad de hábitats. Al contrario, en las partes altas el $\beta$ sim es menor, llegando a ser nulo, y paralelamente el $\beta$ nes aumenta, llegando a explicar todo el cambio de diversidad beta entre los sitios (Fig. 4).

\section{DISCUSIÓN}

Este estudio aporta evidencia sobre el patrón altitudinal de riqueza de peces tropicales

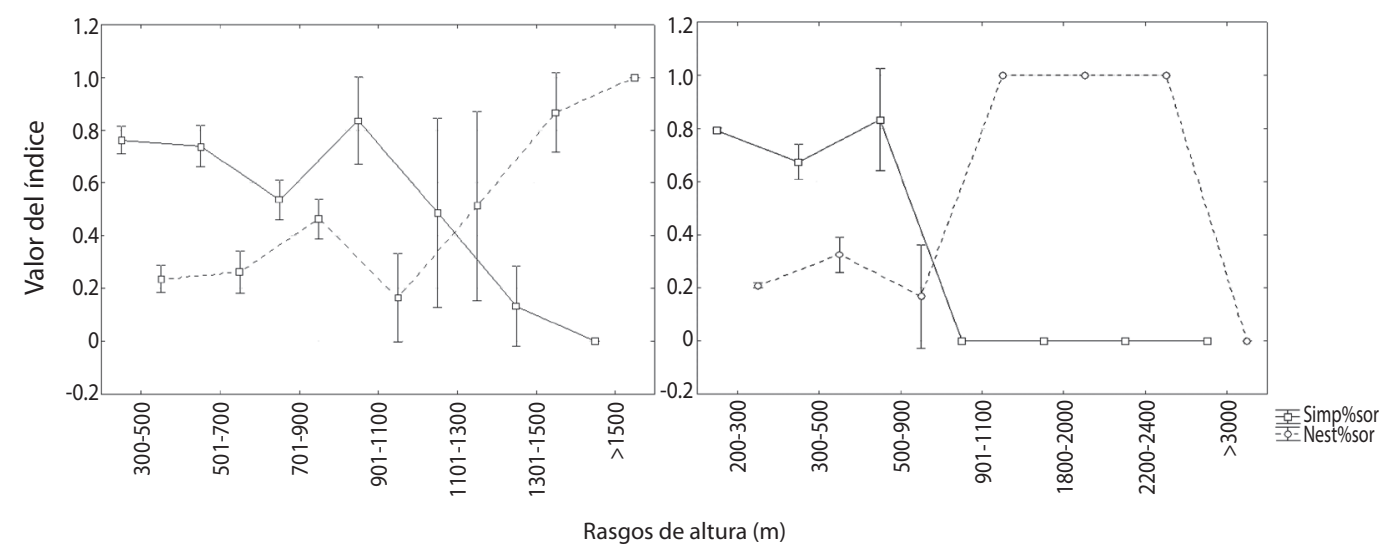

Fig. 4. Índices $\beta$ sim y $\beta$ nes, en porcentaje del índice de disimilitud total ( $\beta$ sor). Izquierda, en la cuenca del río Alto Beni; Derecha, en la cuenca del río Espíritu Santo.

Fig. 4. $\beta$ sim and $\beta$ nes values (in percentage of $\beta$ sor) along the altitudinal gradient. Upper Beni (left) and Espíritu Santo (right) drainages. 
y de la relación significativa de la riqueza de especies con algunas variables ambientales. A nivel general, corroboramos el patrón de disminución gradual de la riqueza de especies con el incremento de la altura encontrado anteriormente tanto en la región tropical (Jaramillo-Villa et al., 2010; Lujan et al., 2013; Miranda-Chumacero, 2006; Pouilly et al., 2006) como en zonas templadas (Rahel, 1991). Esta relación lineal entre riqueza y altura no puede ser un artificio ligado a la escala espacial del estudio (Nogués-Bravo et al. 2008) puesto que se analizó la totalidad del gradiente de altura, por lo menos en lo que se refiere a una de las dos cuencas (i.e. intervalo altitudinal de 200 a $4000 \mathrm{~m}$ en la cuenca del río Espíritu Santo). Hemos notado también que había una diferencia de riqueza local entre las dos cuencas. Como existe normalmente una relación positiva entre la riqueza local y riqueza regional de peces de ríos (Oberdorff et al., 2011) es probable que esta diferencia observada sea consecuencia de una diferencia de riqueza de los colonizadores potenciales entre las dos cuencas.

De acuerdo al presente estudio la distribución de las especies sigue un patrón de tipo 'Rapoport' (Rapoport, 1982) que, aplicada al gradiente altitudinal, estipula que (1) la amplitud del intervalo de distribución de las especies aumenta con la altura, (2) que este patrón es normalmente asociado a una disminución de la riqueza taxonómica con la altura, y (3) que las comunidades con riqueza baja están anidadas en las comunidades con riqueza alta (Beketov, 2009). Estas tres condiciones fueron encontradas en nuestro estudio. Este tipo de distribución en el gradiente altitudinal ya fue registrado en insectos del orden Ephemeroptera, mostrando que a mayores altitudes se encuentran solo algunas de las muchas especies registradas en la parte baja del gradiente (Beketov, 2009). En este estudio, solo las dos especies que se encuentran en todo el gradiente fueron encontradas a más de $2000 \mathrm{~m}$ de altitud (Trichomycterus sp. y Astroblepus sp.). Así mismo, el análisis de las densidades de peces a lo largo del gradiente altitudinal prueba que para la mayoría de las especies, la fuente de colonizadores potenciales está en las partes bajas, con cada vez menos individuos a medida que aumenta la dificultad de dispersión a mayor altura. Las cuatro especies que no siguen este patrón son Astroblepus sp., Trichomycterus sp., Hemibrycon beni y Bryconacidnus ellisi (correlaciones positivas Apéndice 7), lo que sugiere una adaptación de estas especies a las partes altas del gradiente, con un probable flujo de dispersión inverso.

A continuación, en el marco teórico establecido por las cuatro hipótesis, discutimos sobre cada explicación potencial de los patrones altitudinales puestos en evidencia.

Aunque hemos probado parcialmente la hipótesis de la energía disponible (1) (i.e. recursos disponibles) no parece ser un factor limitante para la colonización de las especies de peces en el gradiente altitudinal, debido a que las especies capturadas son principalmente invertívoras y que la densidad de macro-invertebrados tiende a incrementarse con la altura.

Hemos tomado la temperatura del agua como una medida de evaluación de la hipótesis del rigor climático (2), la que determina los límites fisiológicos de las especies (Hipótesis de los limites fisiológicos de Turner et al., 1987). La predicción de esta hipótesis es que si la temperatura es el principal factor determinante de la variación de riqueza con la altura, el patrón predicho es una disminución lineal de la riqueza con el aumento de altura (McCain, 2007). Confirmamos la hipótesis del rigor climático, ya que encontramos un patrón de disminución gradual de la riqueza de especies con el incremento de la altura y que la temperatura del agua fue el factor explicativo más poderoso del modelo estadístico de riqueza, explicando el $66 \%$ de la variación de riqueza para las dos cuencas. Siendo los peces animales ectotermos, casi todos los aspectos de su ecología están asociados a la temperatura (metabolismo, desove, crecimiento, etc.), por lo que no es sorprendente que la temperatura sea un factor dominante de la distribución de la riqueza de especies a lo largo del gradiente de altura (Jacobsen, 2008; Jaramillo-Villa et al., 2010). 
La hipótesis de la diversidad de hábitats (3) (Williams, 1964) aplicada a este estudio relaciona el aumento de la altura con una disminución del tamaño y de la diversidad de hábitats disponibles para los peces. Entre las tres variables seleccionadas (i.e. ancho de río, profundidad y distancia a la fuente de colonización) solo la variable "ancho del río" entra de manera significativa en el modelo, explicando el $13 \%$ de la variación total de la riqueza, esta variable puede ser usada como una medida sintética del tamaño y la diversidad de hábitats disponibles (Tejerina-Garro et al., 2005). Sin embargo, como esta variable no tiene correlación significativa con la altura $(\mathrm{r}=-0.20 ; \mathrm{p}>$ 0.05 ) parece actuar solo al nivel local (del sitio) y no a la escala del gradiente altitudinal. Así mismo, si la hipótesis de diversidad de hábitats fuera válida la variable distancia a la fuente de colonización (medida inversa a la distancia a la fuente) debería entrar en el modelo de manera significativa y negativa, debido a que es una medida indirecta del aumento del volumen de agua disponible a lo largo del gradiente longitudinal. Por tanto, podemos sugerir que esta hipótesis no tiene validez para los peces. En cambio, la variable relacionada a un gradiente de severidad en las condiciones físicas (4), i.e. pendiente máxima en el río principal abajo del sitio, explica $10 \%$ de la variación total de riqueza en el modelo. Las fuertes pendientes del río principal a lo largo del gradiente altitudinal, generan barreras físicas que actúan como un filtro severo de colonización de las especies abajo/arriba. Las diferencias de variaciones de los índices de disimilitud de las comunidades entre las dos cuencas analizadas corroboran esta hipótesis. En efecto, si bien los valores de los índices siguen el mismo patrón en las dos cuencas, en la cuenca del río Alto Beni los valores de $\beta$ sim son mayores a los de $\beta$ nes hasta el rango de altura de $1100-1300 \mathrm{~m}$, mientras que en los arroyos que desembocan en el río Espíritu Santo el rango de altura donde el $\beta$ sim es superado por el $\beta$ nes es entre 900 a $1100 \mathrm{~m}$. Estos cambios son paulatinos en los arroyos de la cuenca del río Alto Beni y bruscos en los del Espíritu Santo (Fig. 4), lo cual podría explicarse por los cambios de pendientes máximas en el río principal, que son mucho más abruptos en el río Espíritu Santo en relación al Alto Beni (Apéndice 8).

Aunque los resultados parecen claros existen al menos dos limitaciones en nuestro estudio. La primera es que los muestreos se realizaron en una sola época (época seca), por lo que sería importante realizar muestreos en la época de lluvias para confirmar los patrones encontrados, sin embargo debemos mencionar que los muestreos en época de lluvias podrían subestimar las poblaciones de peces debido a los grandes volúmenes de agua. La segunda es que no se tomaron en cuenta variables como el sustrato y la velocidad de la corriente que se sabe que actúan sobre la distribución local de las comunidades de peces (Tejerina-Garro et al., 2005). Sin embargo, estas variables no deberían influir de gran manera ya que en los arroyos andinos el sustrato y la velocidad son generalmente homogéneos (sustrato grueso y velocidades altas) (Jacobsen, 2008). Así mismo, en este estudio en la selección de los sitios se eligieron aquellos con rangos de velocidad y sustrato comparables.

En síntesis, la desaparición gradual de especies con el incremento de la altura que se muestra en nuestros resultados, puede explicarse principalmente por dos hipótesis: (1) la hipótesis de rigor climático y (2) la hipótesis de severidad de las condiciones físicas del hábitat. Tomando en cuenta factores asociados a estas dos hipótesis, y sin necesidad de referirse explícitamente a la altura, se puede explicar la mayor parte de las variaciones de la riqueza de peces a lo largo del gradiente altitudinal de las dos cuencas estudiadas. El próximo paso será determinar si el patrón encontrado en este estudio se verifica en otras cuencas tanto en zonas tropicales como templadas. Así mismo, se podría analizar los aspectos funcionales como la ecomorfología de las especies, ya que es claro que ciertas características morfológicas permiten que algunos grupos superen las barreras y colonicen diferentes zonas en el gradiente altitudinal. 


\section{AGRADECIMIENTOS}

Los datos de este estudio fueron tomados en colaboración entre el proyecto $\mathrm{AD}-\mathrm{BOL}$ F57 de Naciones Unidas, la ULRA y el Institut de Recherche pour le Développement (IRD). Un agradecimiento especial a Pablo Tedesco y Danny Rejas por la revisión editorial y valiosos comentarios de este manuscrito. Esta investigación es parte del trabajo de tesis de doctorado de E. De La Barra, financiado a través de una beca doctoral por el IRD.

\section{RESUMEN}

Los gradientes altitudinales representan un sistema apropiado para evaluar si existe una relación general entre los patrones de riqueza, variables ambientales, y los procesos ecológicos que determinan cuantas especies co-ocurren en un área dada. En cuanto a los peces de agua dulce en arroyos de montaña la relación prevalente es un decrecimiento monotónico en riqueza de especies con la altura. El objetivo de este estudio es evaluar cuatro hipótesis que pueden explicar la relación negativa entre la riqueza local de especies de peces y la altura, 1) hipótesis de la disminución de la energía disponible 2) hipótesis del aumento del rigor climático 3) hipótesis de la diversidad de hábitats e 4) hipótesis de la severidad de las condiciones físicas del hábitat que se presentan río arriba, relacionada a las dificultades para colonizar las partes altas de los ríos. Se recolectaron peces y macro-invertebrados en 83 sitios entre 200 a 4000 m en dos cuencas en la Amazonía boliviana. La primera hipótesis fue evaluada mediante regresiones entre la densidad de macro-invertebrados, los peces invertívoros y la altura. Para evaluar la segunda y tercera hipótesis se realizó un análisis de regresión múltiple (GLM), entre la riqueza y varios factores locales y regionales. Se analizó también la disimilitud de los ensamblajes de peces sobre el gradiente altitudinal utilizando los índices $\beta$ sim y $\beta$ nes. Se encontró que la riqueza de peces disminuye de manera lineal con el aumento de la altura. La densidad de macroinvertebrados aumenta con la altura, contrariamente a la riqueza de peces invertívoros, sugiriendo que la energía no constituye un factor limitante para la colonización de las especies de peces en zonas altas. El GLM explicó un $86 \%$ de la variación total de la riqueza, con un aporte significativo de la temperatura del agua, las pendientes máximas en el río principal y el ancho de río. El $\beta$ sim presentó valores altos en niveles de altura bajos, mostrando mayor recambio de especies entre los sitios cercanos a la fuente de colonización. Inversamente, el $\beta$ nes mostró valores mayores en las partes altas, correspondientes a cambios por pérdida de especies. Estos resultados sugieren que el rigor climático junto con la severidad física del hábitat crean barreras a la colonización y explican los cambios de riqueza en el gradiente altitudinal.

Palabras clave: riqueza de especies, Amazonía boliviana, arroyos de montaña, máximas pendientes en el río principal.

\section{REFERENCIAS}

Baselga, A. (2010). Partitioning the turnover and nestedness components of beta diversity. Global Ecology and Biogeography, 19, 134-143.

Beketov, M. A. (2009). The Rapoport effect is detected in a river system and is based on nested organisation. Global Ecology and Biogeography, 18, 498-506.

Cattaneo, F., Hugueny, B., \& Lamouroux, N. (2003). Synchrony in brown trout, Salmo trutta, population dynamics: a "Moran effect" on early-life stages. Oikos, 100, 43-54.

Eigenmann, C. H. (1918). The Pygiidae, a family of South American catfishes. Memoirs of the Carnegie Museum, 7, 259-398.

Géry, J. (1977). Characoids of the World. Neptune city: T.f.h. Publications, Inc. Ltd.

Ibañez, C., Tedesco, P., Bigorne, R., Hugueny, B., Pouilly, M., Zepita, C., Zubieta, J., \& Oberdorff, T. (2007). Dietary-morphological relationships in fish assemblages of small forested streams in the Bolivian Amazon. Aquatic Living Resources, 20, 131-142.

Jacobsen, D. (2008). Tropical high-altitude streams. En D. Dudgeon (Ed.), Tropical Stream Ecology (pp. 219256). London, UK: Academic Press.

Jaramillo-Villa, U., Maldonado-Ocampo, J. A., \& Escobar, F. (2010). Altitudinal variation in fish assemblage diversity in streams of the central Andes of Colombia. Journal of Fish Biology, 76, 2401-2417.

Lomolino, M. (2001). Elevation gradients of species-density: historical and prospective views. Global Ecology \& Biogeography, 10, 3-13.

Lujan, N. K., Jacobsen, D., Winemiller, K. O., Meza, V. V., Rimarachín, V., \& Arana, M. J. (2013). Aquatic community structure across an Andes-to-Amazon fluvial gradient. Journal of Biogeography, 40, 1715-1728.

McCain, C. M. (2007). Could temperature and water availability drive elevational species richness? A global case study for bats. Global Ecology and Biogeography, 16, 1-13.

Miranda-Chumacero, G. (2006). Distribución altitudinal, abundancia relativa y densidad de peces en el Río Huarinilla y sus tributarios (Cotapata, Bolivia). Ecología en Bolivia, 41, 79-93.

Moya, N., Hughes, R. M., Dominguez, E., Gibon, F. M., Goitia, E., \& Oberdorff, T. (2011). 
Macroinvertebrate-based multimetric predictive models for evaluating the human impact on biotic condition of Bolivian streams. Ecological Indicators, $11,840-847$.

Navarro, G., \& Maldonado, M. (2002). Geografia ecológica de Bolivia. Vegetación y ambientes acuáticos. Cochabamba, Bolivia: Centro de Ecología Simón I. Patiño.

Nogués-Bravo, D., Araujo, M. B., Romdal, T., \& Rahbek, C. (2008). Scale effects and human impact in the elevational species richness gradients. Nature, 453, 216-220.

Oberdorff, T., Tedesco, P. A., Hugueny, B., Leprieur, F., Beauchard, O., Brosse, S., \& Dürr, H. H. (2011). Global and regional patterns in riverine fish species richness: A review. International Journal of Ecology, Volume 2011, Article ID 967631, 1-12.

Petts, G., \& Calow, P. (1996). River Biota: Diversity and Dynamics. Oxford: Blackwell Science.

Pearson, N. E. (1924). The fishes of the eastern slope of the Andes. I. The fishes of the Rio Beni basin, Bolivia, collected by the Mulford Expedition. Indiana: Indiana University Studies.

Pouilly, M., Barrera, S., \& Rosales, C. (2006). Changes of taxonomic and trophic structure of fish assemblages along an environmental gradient in the Upper Beni watershed (Bolivia). Journal of Fish Biology, 68, 137-156.

Rahbek, C. (1995). The elevational gradient of species richness: a uniform pattern? Ecography, 18, 200-205.
Rahel, F. (1991). Fish Assemblager and Habitat Gradients in a Rocky Mountain-Great Plains Stream: Biotic Zonatión and Additive Patterns of Community Change. Transactions of the American Fisheries Society, 120, 319-332.

Rapoport, E. (1982). Areography. Geographical Strategies of Species. Oxford: Pergamon Press.

Tedesco, P., Ibañez, C., Moya, N., Bigorne, R., Camacho, J., Goitia, E., Hugueny, B., Maldonado, M., Rivero, M., Tomanová, S., Zubieta, J., \& Oberdorff, T. (2007). Local-scale species-energy relationships in fish assemblages of some forested streams of the Bolivian Amazon. Comptes Rendus Biologies, 330, 255-264.

Tejerina-Garro, F., Maldonado, M., Ibañez, C., Pont, D., Roset, N., \& Oberdorff, T. (2005). Effects of natural and anthropogenic environmental changes on riverine fish assemblages: a framework for ecological assessment of rivers. Brazilian Archives of Biology and Technology, 48, 91-108.

Turner, J. R. G., Gatehouse, C. M., \& Corey, C. A. (1987). Does solar energy control organic diversity? Butterflies, moths and the British climate. Oikos, 48, 195-205.

Whittingham, M. J., Stephens, P. A., Bradbury, R. B., \& Freckleton, R. B. (2006). Why do we still use stepwise modelling in ecology and behaviour? Journal of Animal Ecology, 75, 1182-1189.

Williams, C. B. (1964). Patterns in the balance of nature. London: Academic Press.

Wright, D. (1983). Species-energy theory: an extension of species-area theory. Oikos, 41, 496-506. 
APÉNDICE 1

Datos usados para el análisis de regresión múltiple y ubicación geográfica de los sitios

APPENDIX 1

Data used for the multiple regression analysis and geographical position of sites

\begin{tabular}{|c|c|c|c|c|c|c|c|c|c|c|}
\hline Cuenca & Código & Y & $X$ & Riqueza & $\begin{array}{l}\text { Altura } \\
(\mathrm{m})\end{array}$ & $\begin{array}{c}\text { Ancho } \\
(\mathrm{m})\end{array}$ & $\begin{array}{l}\text { Prof. } \\
(\mathrm{cm})\end{array}$ & $\begin{array}{c}\text { Temp. } \\
\left({ }^{\circ} \mathrm{C}\right)\end{array}$ & $\begin{array}{l}\text { Distancia a la } \\
\text { fuente de } \\
\text { colonización (m) }\end{array}$ & PMRP \\
\hline E. Santo & 101BB & -17.066316 & -65.487335 & 9 & 462 & 5.3 & 16.9 & 22.67 & 159365 & 0.020 \\
\hline E. Santo & $102 \mathrm{E}$ & -16.845942 & -65.332116 & 18 & 218 & 6.5 & 32.1 & 24.83 & 114878 & 0.003 \\
\hline E. Santo & $200 \mathrm{~B}$ & -16.994060 & -65.333645 & 17 & 250 & 6.1 & 25.3 & 24.54 & 151971 & 0.003 \\
\hline E. Santo & $200 \mathrm{~F}$ & -16.976110 & -65.361294 & 11 & 286 & 6.9 & 24.2 & 24.21 & 144952 & 0.003 \\
\hline E. Santo & $316 \mathrm{~B}$ & -17.010812 & -65.450138 & 20 & 333 & 6.9 & 22.1 & 23.80 & 151435 & 0.040 \\
\hline E. Santo & $319 \mathrm{C}$ & -17.003206 & -65.583122 & 6 & 427 & 4.7 & 21.6 & 22.98 & 169305 & 0.030 \\
\hline E. Santo & MAB01 & -17.233861 & -66.075194 & 1 & 4000 & 2.2 & 17.5 & 4.85 & 269517 & 0.080 \\
\hline E. Santo & MAB02 & -17.268618 & -65.880052 & 1 & 3251 & 5.5 & 13.5 & 6.53 & 228443 & 0.110 \\
\hline E. Santo & MAB03 & -17.370483 & -65.821870 & 1 & 3277 & 4.0 & 9.0 & 6.46 & 233573 & 0.110 \\
\hline E. Santo & MAB04 & -17.133023 & -66.144139 & 2 & 3708 & 6.0 & 16.5 & 5.37 & 267539 & 0.110 \\
\hline E. Santo & MAB05 & -17.192361 & -65.798702 & 2 & 1852 & 7.8 & 22.7 & 12.69 & 225228 & 0.110 \\
\hline E. Santo & MAB06 & -17.212762 & -65.825738 & 2 & 1971 & 4.0 & 40.0 & 12.01 & 240128 & 0.110 \\
\hline E. Santo & MAB07 & -17.203677 & -65.857144 & 1 & 2320 & 6.0 & 24.6 & 10.19 & 222379 & 0.110 \\
\hline E. Santo & MAB08 & -17.211638 & -65.820238 & 1 & 1892 & 9.1 & 51.5 & 12.46 & 219290 & 0.110 \\
\hline E. Santo & MAB09 & -17.187972 & -65.754755 & 2 & 1914 & 4.2 & 24.5 & 12.33 & 215930 & 0.110 \\
\hline E. Santo & MAB10 & -17.185262 & -65.746250 & 3 & 1877 & 3.0 & 30.8 & 12.54 & 214914 & 0.110 \\
\hline E. Santo & MAB11 & -17.133842 & -65.701930 & 3 & 1120 & 4.3 & 32.0 & 17.46 & 196851 & 0.090 \\
\hline E. Santo & MAB12 & -17.060425 & -65.646001 & 8 & 628 & 10.9 & 41.1 & 21.28 & 186000 & 0.070 \\
\hline E. Santo & MAB13 & -16.998287 & -65.598468 & 7 & 462 & 3.6 & 26.8 & 22.67 & 172358 & 0.040 \\
\hline E. Santo & MAB14 & -17.003206 & -65.583122 & 4 & 445 & 3.8 & 17.3 & 22.82 & 169305 & 0.030 \\
\hline E. Santo & MAB15 & -17.022063 & -65.523561 & 7 & 361 & 6.7 & 33.3 & 23.55 & 160614 & 0.030 \\
\hline E. Santo & MAB16 & -17.010770 & -65.450231 & 9 & 331 & 8.6 & 47.2 & 23.82 & 151440 & 0.040 \\
\hline E. Santo & MAB17 & -17.133842 & -65.701930 & 1 & 975 & 1.5 & 24.3 & 18.54 & 196851 & 0.090 \\
\hline E. Santo & MAB18 & -17.015849 & -65.652686 & 6 & 600 & 9.3 & 18.0 & 21.51 & 180539 & 0.080 \\
\hline E. Santo & MAB19 & -17.015008 & -65.548269 & 9 & 389 & 3.2 & 35.0 & 23.31 & 163221 & 0.060 \\
\hline E. Santo & MAB20 & -17.087436 & -65.675690 & 5 & 811 & 3.0 & 10.0 & 19.80 & 190612 & 0.090 \\
\hline E. Santo & MAB21 & -17.007969 & -65.558547 & 7 & 394 & 7.9 & 18.0 & 23.26 & 165292 & 0.040 \\
\hline E. Santo & MAB22 & -17.060580 & -65.482959 & 8 & 425 & 9.7 & 44.8 & 22.99 & 159312 & 0.020 \\
\hline E. Santo & MAB23 & -17.004382 & -65.639527 & 5 & 480 & 2.9 & 24.9 & 22.52 & 177130 & 0.050 \\
\hline Alto Beni & $11-30$ & -15.551749 & -67.305848 & 13 & 460 & 2.5 & 19.2 & 22.69 & 174438 & 0.010 \\
\hline Alto Beni & $11-35$ & -16.067253 & -67.158944 & 4 & 743 & 3.7 & 13.4 & 20.34 & 261226 & 0.020 \\
\hline Alto Beni & $11-36$ & -16.068833 & -67.114628 & 3 & 761 & 5.1 & 32.4 & 20.20 & 262627 & 0.020 \\
\hline Alto Beni & $11-46$ & -15.340906 & -67.183842 & 14 & 629 & 7.5 & 33.1 & 21.27 & 184861 & 0.001 \\
\hline Alto Beni & $11-47$ & -15.348054 & -67.176023 & 10 & 610 & 4.2 & 16.7 & 21.43 & 186352 & 0.001 \\
\hline Alto Beni & $11-48$ & -15.356802 & -67.163961 & 10 & 714 & 4.1 & 26.4 & 20.58 & 188278 & 0.001 \\
\hline Alto Beni & $11-50$ & -15.412237 & -67.334032 & 17 & 430 & 3.8 & 23.7 & 22.95 & 152845 & 0.001 \\
\hline Alto Beni & $11-61$ & -15.760824 & -67.062462 & 8 & 555 & 2.0 & 17.7 & 21.89 & 215042 & 0.002 \\
\hline Alto Beni & $11-03$ & -15.913369 & -67.235418 & 9 & 563 & 5.9 & 26.8 & 21.82 & 235997 & 0.050 \\
\hline Alto Beni & $11-04$ & -15.941461 & -67.216463 & 9 & 594 & 5.1 & 20.1 & 21.56 & 240449 & 0.010 \\
\hline Alto Beni & $11-14$ & -16.252853 & -67.116542 & 7 & 810 & 3.4 & 18.3 & 19.81 & 288930 & 0.010 \\
\hline Alto Beni & $11-22$ & -16.262617 & -67.379742 & 18 & 406 & 4.7 & 18.0 & 23.16 & 321022 & 0.010 \\
\hline Alto Beni & $11-25$ & -15.434809 & -67.453351 & 11 & 300 & 2.0 & 32.8 & 24.09 & 143937 & 0.001 \\
\hline
\end{tabular}


APÉNDICE 1 (Continuación) / APPENDIX 1 (Continued)

\begin{tabular}{|c|c|c|c|c|c|c|c|c|c|c|}
\hline Cuenca & Código & $\mathrm{Y}$ & $\mathrm{X}$ & Riqueza & $\begin{array}{c}\text { Altura } \\
(\mathrm{m})\end{array}$ & $\begin{array}{c}\text { Ancho } \\
\text { (m) }\end{array}$ & $\begin{array}{l}\text { Prof. } \\
(\mathrm{cm})\end{array}$ & $\begin{array}{l}\text { Temp. } \\
\left({ }^{\circ} \mathrm{C}\right)\end{array}$ & $\begin{array}{l}\text { Distancia a la } \\
\text { fuente de } \\
\text { colonización (m) }\end{array}$ & PMRP \\
\hline Alto Beni & A 01 & -16.469055 & -67.482885 & 2 & 1529 & 6.5 & 19.1 & 14.66 & 346138 & 0.010 \\
\hline Alto Beni & A 02 & -16.428563 & -67.467272 & 4 & 1248 & 4.0 & 24.3 & 16.55 & 341065 & 0.010 \\
\hline Alto Beni & A 03 & -16.420174 & -67.543183 & 3 & 1545 & 3.2 & 9.9 & 14.56 & 346578 & 0.010 \\
\hline Alto Beni & A 04 & -16.384676 & -67.518787 & 4 & 1453 & 2.0 & 13.0 & 15.16 & 345915 & 0.010 \\
\hline Alto Beni & A 05 & -16.328365 & -67.555536 & 4 & 1254 & 1.9 & 14.9 & 16.51 & 352197 & 0.100 \\
\hline Alto Beni & A 06 & -16.339105 & -67.585043 & 8 & 1096 & 2.0 & 12.2 & 17.64 & 352523 & 0.010 \\
\hline Alto Beni & A 07 & -16.283702 & -67.640728 & 4 & 1449 & 3.6 & 16.5 & 15.18 & 354703 & 0.020 \\
\hline Alto Beni & A 08 & -16.260728 & -67.643167 & 3 & 1524 & 2.2 & 10.7 & 14.69 & 353619 & 0.020 \\
\hline Alto Beni & A 11 & -15.937407 & -67.467769 & 4 & 849 & 1.9 & 12.9 & 19.51 & 249027 & 0.010 \\
\hline Alto Beni & A 12 & -15.937328 & -67.474589 & 12 & 806 & 4.3 & 20.3 & 19.84 & 247649 & 0.010 \\
\hline Alto Beni & A 13 & -15.805305 & -67.490144 & 5 & 758 & 2.0 & 9.7 & 20.22 & 239092 & 0.010 \\
\hline Alto Beni & A 14 & -15.793384 & -67.512317 & 8 & 728 & 3.0 & 21.6 & 20.46 & 240215 & 0.010 \\
\hline Alto Beni & A 16 & -15.731600 & -67.512310 & 5 & 882 & 2.6 & 13.2 & 19.25 & 253852 & 0.010 \\
\hline Alto Beni & A 17 & -15.719291 & -67.519127 & 8 & 892 & 3.8 & 21.1 & 19.17 & 255460 & 0.010 \\
\hline Alto Beni & A 18 & -15.533449 & -67.386259 & 13 & 389 & 4.7 & 28.4 & 23.31 & 162395 & 0.001 \\
\hline Alto Beni & A 19 & -15.505486 & -67.422399 & 11 & 406 & 2.3 & 17.2 & 23.16 & 155182 & 0.001 \\
\hline Alto Beni & A 20 & -15.490421 & -67.432719 & 16 & 376 & 4.5 & 28.3 & 23.42 & 153781 & 0.005 \\
\hline Alto Beni & A 21 & -15.454633 & -67.448466 & 15 & 387 & 2.7 & 20.2 & 23.32 & 148325 & 0.002 \\
\hline Alto Beni & A 22 & -15.642238 & -67.160558 & 16 & 422 & 4.7 & 18.9 & 23.02 & 194714 & 0.010 \\
\hline Alto Beni & A 23 & -15.674022 & -67.130995 & 19 & 450 & 7.1 & 17.4 & 22.78 & 199939 & 0.002 \\
\hline Alto Beni & A 24 & -15.731651 & -67.049740 & 10 & 483 & 5.9 & 15.8 & 22.49 & 213095 & 0.002 \\
\hline Alto Beni & A 25 & -16.482216 & -67.374614 & 4 & 1340 & 3.7 & 16.3 & 15.92 & 342113 & 0.010 \\
\hline Alto Beni & A 26 & -16.597569 & -67.292400 & 8 & 1163 & 6.7 & 20.6 & 17.15 & 350009 & 0.010 \\
\hline Alto Beni & A 27 & -16.758185 & -67.233333 & 2 & 1986 & 2.4 & 11.5 & 11.93 & 372771 & 0.010 \\
\hline Alto Beni & A 28 & -16.751512 & -67.229998 & 2 & 1905 & 4.7 & 13.0 & 12.38 & 372400 & 0.010 \\
\hline Alto Beni & A 31 & -16.702898 & -67.153885 & 3 & 1409 & 2.7 & 16.9 & 15.45 & 360418 & 0.010 \\
\hline Alto Beni & A 33 & -16.702898 & -67.153885 & 2 & 1470 & 2.2 & 7.9 & 15.05 & 360418 & 0.010 \\
\hline Alto Beni & A 38 & -16.400829 & -67.633630 & 2 & 1253 & 2.1 & 11.2 & 16.52 & 362590 & 0.020 \\
\hline Alto Beni & A 39 & -16.417256 & -67.698434 & 2 & 1348 & 3.4 & 19.7 & 15.86 & 370028 & 0.020 \\
\hline Alto Beni & A 47 & -15.779049 & -67.444881 & 7 & 866 & 4.0 & 16.2 & 19.37 & 249113 & 0.010 \\
\hline Alto Beni & A 51 & -15.750213 & -67.494790 & 15 & 482 & 6.8 & 18.6 & 22.50 & 251205 & 0.010 \\
\hline Alto Beni & A 52 & -15.819026 & -67.595894 & 8 & 574 & 2.4 & 15.1 & 21.73 & 224037 & 0.010 \\
\hline Alto Beni & A 54 & -16.281061 & -67.651655 & 5 & 1456 & 4.1 & 27.1 & 15.14 & 355880 & 0.020 \\
\hline Alto Beni & A 55 & -16.287864 & -67.573185 & 8 & 1072 & 7.3 & 21.9 & 17.82 & 346915 & 0.020 \\
\hline Alto Beni & A 56 & -16.275425 & -67.549810 & 6 & 1017 & 6.5 & 26.6 & 18.22 & 340923 & 0.020 \\
\hline Alto Beni & A 60 & -15.956755 & -67.195032 & 11 & 629 & 5.7 & 14.3 & 21.27 & 245605 & 0.010 \\
\hline Alto Beni & A 62 & -16.107052 & -67.199270 & 11 & 717 & 4.4 & 21.0 & 20.55 & 267645 & 0.030 \\
\hline Alto Beni & A 63 & -16.163392 & -67.135314 & 6 & 988 & 3.3 & 12.3 & 18.44 & 280348 & 0.010 \\
\hline Alto Beni & A 65 & -16.259116 & -67.248761 & 11 & 785 & 6.3 & 15.5 & 20.01 & 298203 & 0.010 \\
\hline Alto Beni & A 66 & -16.292553 & -67.286442 & 8 & 796 & 3.6 & 10.6 & 19.92 & 305338 & 0.010 \\
\hline Alto Beni & A 73 & -15.831532 & -67.572634 & 16 & 572 & 7.3 & 16.1 & 21.74 & 227022 & 0.001 \\
\hline
\end{tabular}

PMRP: Pendientes máximas en el río principal.

PMRP: Maximum slopes in the river mainstem. 


\section{APÉNDICE 2}

Esquema de la asignación de valores de pendiente en el río principal

\section{APPENDIX 2}

Diagram explaining how maximum slopes in the river mainstem were calculated

(Archivo de imagen "Apéndice 2")

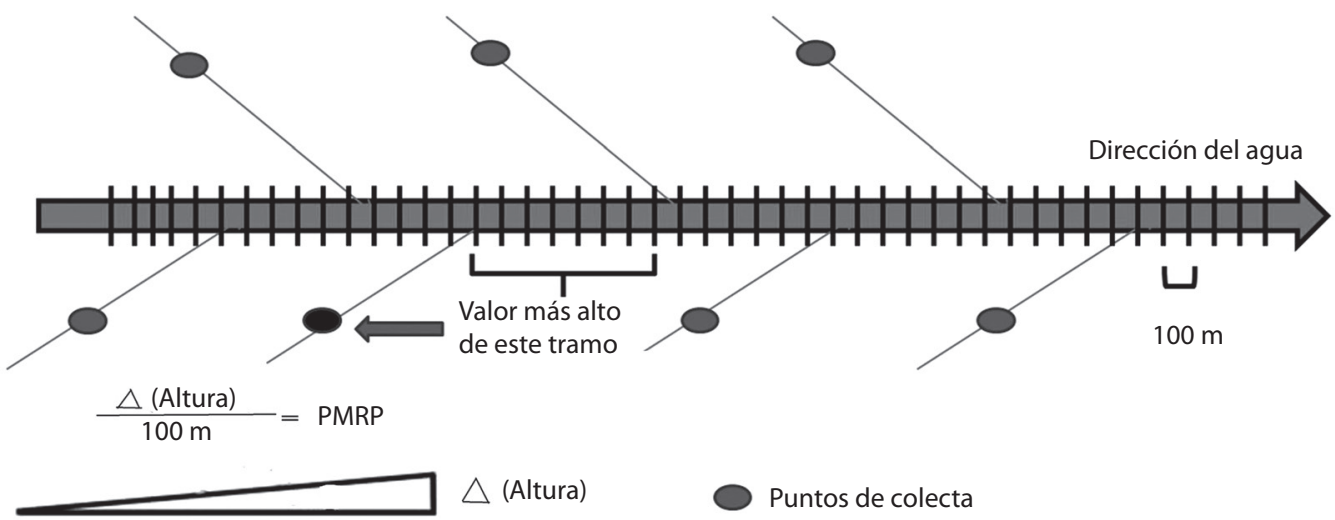

$100 \mathrm{~m}$

APÉNDICE 3

Lista de las especies de peces registradas en las dos cuencas

APPENDIX 3

List of species sampled in the two river basins

\begin{tabular}{|c|c|c|c|c|c|}
\hline \multirow{2}{*}{ Orden } & \multirow{2}{*}{ Familia } & \multirow{2}{*}{ Especie } & \multirow{2}{*}{ Código } & \multicolumn{2}{|c|}{ Cuencas } \\
\hline & & & & Alto Beni & E. Santo \\
\hline \multirow[t]{20}{*}{ Characiformes } & Anostomidae & Leporinus friderici & Lef & $\mathrm{x}$ & \\
\hline & & Leporinus striatus & Les & & $\mathrm{x}$ \\
\hline & Characidae & Acrobrycon ipanquianus & Aci & $\mathrm{x}$ & $\mathrm{x}$ \\
\hline & & Astyanacinus multidens & Asm & $\mathrm{x}$ & $\mathrm{x}$ \\
\hline & & Astyanax abramis & Asa & $\mathrm{x}$ & $\mathrm{x}$ \\
\hline & & Astyanax lineatus & Asl & $\mathrm{x}$ & $\mathrm{x}$ \\
\hline & & Bryconacidnus ellisi & Bre & $\mathrm{x}$ & \\
\hline & & Bryconamericus bolivianus & $\mathrm{Brb}$ & $\mathrm{x}$ & \\
\hline & & Cheirodon sp. & Che & $\mathrm{x}$ & \\
\hline & & Creagrutus beni & $\mathrm{Crb}$ & $\mathrm{x}$ & $\mathrm{x}$ \\
\hline & & Creagrutus pearsoni & Crp & $\mathrm{x}$ & $\mathrm{x}$ \\
\hline & & Gephyrocharax cf. major & Gem & $\mathrm{x}$ & \\
\hline & & Gephyrocharax chaparae & $\mathrm{Gec}$ & & $\mathrm{x}$ \\
\hline & & Hemibrycon beni & Heb & $\mathrm{x}$ & \\
\hline & & Hemibrycon jelskii & Hej & $\mathrm{x}$ & $\mathrm{x}$ \\
\hline & & Hemigrammus lunatus & Hel & & $\mathrm{x}$ \\
\hline & & Hyphessobrycon sp. & Hyp & $\mathrm{x}$ & \\
\hline & & Knodus spp. & Kno & $\mathrm{x}$ & $\mathrm{x}$ \\
\hline & & Moenkhausia sanctaefilomenae & Mos & & $\mathrm{x}$ \\
\hline & & Monotocheirodon paersoni & Mop & $x$ & \\
\hline
\end{tabular}


APÉNDICE 3 (Continuación) / APPENDIX 3 (Continued)

\begin{tabular}{|c|c|c|c|c|c|}
\hline \multirow{2}{*}{ Orden } & \multirow{2}{*}{ Familia } & \multirow{2}{*}{ Especie } & \multirow{2}{*}{ Código } & \multicolumn{2}{|c|}{ Cuencas } \\
\hline & & & & Alto Beni & E. Santo \\
\hline & & Odontostilbe dierythrura & Odd & $\mathrm{x}$ & $\mathrm{X}$ \\
\hline & & Phenacogaster cf. pectinatus & Php & & $\mathrm{x}$ \\
\hline & & Prodontocharax melanotus & Prm & $\mathrm{x}$ & $\mathrm{x}$ \\
\hline & & Serrapinnus sp. & Ser & & $\mathrm{x}$ \\
\hline & & Tyttocharax cf. madeirae & Tym & & $\mathrm{x}$ \\
\hline & Crenuchidae & Characidium bolivianum & $\mathrm{Chb}$ & $\mathrm{x}$ & $\mathrm{x}$ \\
\hline & & Characidium sp.1 & Ch1 & $\mathrm{x}$ & \\
\hline & & Characidium sp. 2 & $\mathrm{Ch} 2$ & & $\mathrm{x}$ \\
\hline & Curimatidae & Steindachnerina dobula & Std & $\mathrm{x}$ & \\
\hline & & Steindachnerina guentheri & Stg & $\mathrm{x}$ & \\
\hline & Erythrinidae & Hoplias gr. malabaricus & Hom & $\mathrm{x}$ & $\mathrm{x}$ \\
\hline & Parodontidae & Parodon sp. & Par & $\mathrm{x}$ & $\mathrm{x}$ \\
\hline & Prochilodontidae & Prochilodus nigricans & Prn & & $\mathrm{x}$ \\
\hline \multirow[t]{4}{*}{ Perciformes } & Cichlidae & Aequidens sp. & Aeq & $\mathrm{x}$ & \\
\hline & & Bujurquina sp. & Buj & $\mathrm{x}$ & $\mathrm{x}$ \\
\hline & & Cichlasoma boliviense & Cib & & $\mathrm{x}$ \\
\hline & & Crenicichla spp. & Cre & $\mathrm{x}$ & $\mathrm{x}$ \\
\hline \multirow[t]{18}{*}{ Siluriformes } & Callichthyidae & Callichthys callichthys & $\mathrm{Cac}$ & $\mathrm{x}$ & \\
\hline & Heptapteridae & Cetopsorhamdia spp. & Cet & $\mathrm{x}$ & $\mathrm{x}$ \\
\hline & & Heptapterus sp. & Hep & & $\mathrm{x}$ \\
\hline & & Imparfinis cf. stictonotus & Ims & & $\mathrm{x}$ \\
\hline & & Pimelodella spp. & Pim & $\mathrm{x}$ & $\mathrm{x}$ \\
\hline & & Rhamdia quelen & Rhq & $\mathrm{x}$ & $\mathrm{x}$ \\
\hline & & Ancistrus spp. & Anc & $\mathrm{x}$ & $\mathrm{x}$ \\
\hline & Loricariidae & Astroblepus spp. & Ast & $\mathrm{x}$ & $\mathrm{x}$ \\
\hline & & Crossoloricaria cf. bahuaja & Cob & & $\mathrm{x}$ \\
\hline & & Farlowella cf. oxyrryncha & Fao & & $\mathrm{x}$ \\
\hline & & Hypostomus bolivianus & Hyb & $\mathrm{x}$ & \\
\hline & & Hypostomus gr. cochliodon & Нyp & & $\mathrm{x}$ \\
\hline & & Hypostomus levis & Hyl & $\mathrm{x}$ & \\
\hline & & Rineloricaria lanceolata & Ril & $\mathrm{x}$ & $\mathrm{x}$ \\
\hline & Pseudopimelodidae & Batrochoglanis raninus & Bar & & $\mathrm{x}$ \\
\hline & & Microglanis cf. poecilus & Mip & & $\mathrm{x}$ \\
\hline & Trichomycteridae & Ituglanis amazonicus & Ita & & $\mathrm{x}$ \\
\hline & & Trichomycterus spp. & Tri & $\mathrm{x}$ & $\mathrm{x}$ \\
\hline Synbranchiformes & Synbranchidae & Synbranchus marmoratus & Sym & $\mathrm{x}$ & $\mathrm{x}$ \\
\hline
\end{tabular}




\section{APÉNDICE 4}

Relación (Lowess con factor de alisamiento $=0.7$ ) independiente entre la riqueza y las principales variables ambientales: pendientes máximas en el río principal (A), temperatura del agua (B) y ancho de río (C)

\section{APPENDIX 4}

Relationships (Lowess smoothing O.7) between local fish species richness and the three major environmental variables: maximum slopes in the river mainstem (A), water temperature (B) and mean stream width (C)

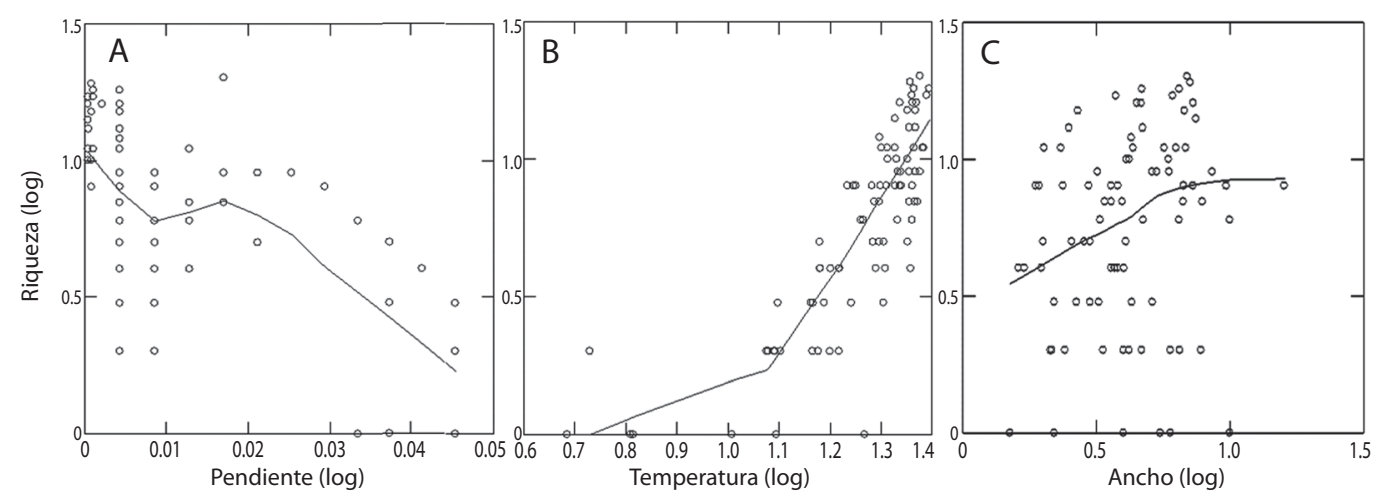

\section{APÉNDICE 5}

Relación entre la altura y (A) la densidad de macro-invertebrados (lineal), y (B) la riqueza de peces invertívoros (Lowess con factor de alisamiento $=0.7$ )

\section{APPENDIX 5}

Relationships between altitude and (A) local macro-invertebrates density (linear) and (B) local invertivorous fish species richness (Lowess smoothing O.7)
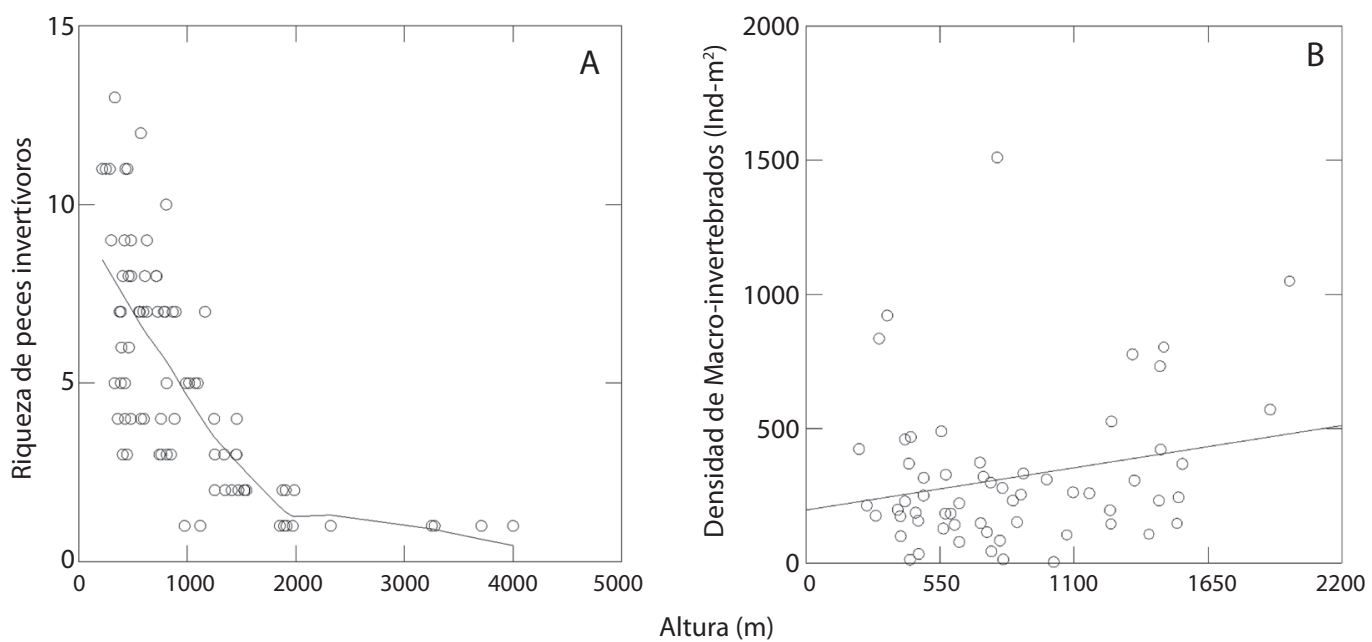
APÉNDICE 6

Esquema de las pendientes máximas en el río principal como barreras a la colonización de los peces y su relación con el gradiente altitudinal

\section{APPENDIX 6}

Diagram explaining how maximum slopes in the river mainstem act as barriers to colonization for fishes along the altitudinal gradient

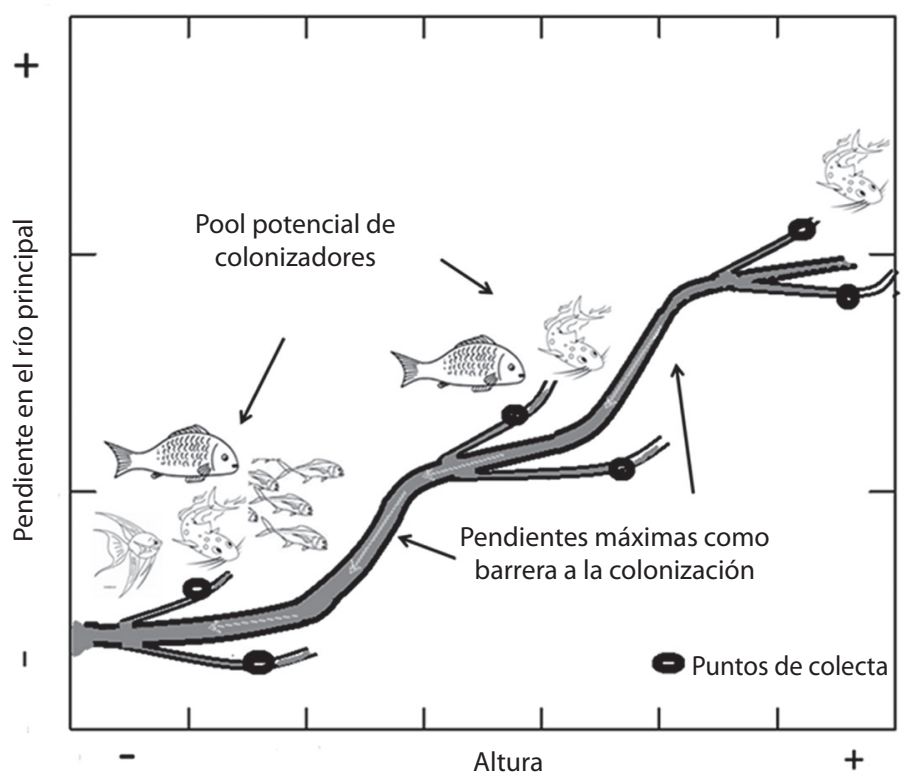

APÉNDICE 7

Correlación entre las densidades locales de cada especie y la altura

\section{APPENDIX 7}

Correlation between each fish species local density and altitude

\begin{tabular}{lc}
\multicolumn{1}{c}{ Especies } & Correlación Spp/Altura \\
Acrobrycon ipanquianus & -0.02 \\
Aequidens sp. & -0.15 \\
Ancistrus sp. & -0.01 \\
Astroblepus sp.* & 0.50 \\
Astyanacinus multidens & -0.21 \\
Astyanax abramis & -0.41 \\
Astyanax lineatus & -0.33 \\
Bryconacidnus ellisi* & 0.08 \\
Bryconamericus bolivianus & -0.21 \\
Bujurquina sp. & -0.19 \\
Callichthys callichthys & -0.11 \\
Cetopsorhamdia sp. & -0.20 \\
Characidae sp. & -0.11 \\
Characidium bolivianum & -0.33 \\
Characidium sp. & -0.31 \\
\hline
\end{tabular}

\begin{tabular}{lc}
\multicolumn{1}{c}{ Especies } & Correlación Spp/Altura \\
Characidium sp. & -0.27 \\
Cheirodon sp. & -0.13 \\
Cichlasoma boliviense & -0.19 \\
Creagrutus beni & -0.40 \\
Creagrutus pearsoni & -0.18 \\
Crenicichla sp. & -0.37 \\
Crossoloricaria cf. bahuaja & -0.20 \\
Farlowella cf oxyrryncha & -0.11 \\
Gephyrocharax cf. major & -0.16 \\
Gephyrocharax chaparae & -0.19 \\
Hemibrycon beni* & 0.11 \\
Hemibrycon jelskii & -0.31 \\
Hemigrammus lunatus & -0.18 \\
Hoplias gr. malabaricus & -0.29 \\
Hyphessobrycon sp. & -0.27 \\
\hline
\end{tabular}


APÉNDICE 7 (Continuación) / APPENDIX 7 (Continued)

\begin{tabular}{lc}
\multicolumn{1}{c}{ Especies } & Correlación Spp/Altura \\
Hypostomus bolivianus & -0.27 \\
Hypostomus gr. cochliodon & -0.17 \\
Hypostomus levis & -0.26 \\
Imparfinis cf. Stictonotus & -0.27 \\
Ituglanis amazonicus & -0.15 \\
Knodus sp. & -0.27 \\
Leporinus friderici & -0.11 \\
Leporinus striatus & -0.27 \\
Microglanis sp. & -0.19 \\
Moenkhausia sanctaefilomenae & -0.18 \\
Monotocheirodon pearsoni & -0.08 \\
Odontostilbe dierythrura & -0.38 \\
Parodon sp. & -0.27 \\
\hline
\end{tabular}

\begin{tabular}{lc}
\multicolumn{1}{c}{ Especies } & Correlación Spp/Altura \\
Phenacogaster cf. pectinatus & -0.19 \\
Pimelodella sp. & -0.33 \\
Prochilodus nigricans & -0.22 \\
Prodontocharax melanotus & -0.10 \\
Rhamdia quelen & -0.33 \\
Rineloricaria lanceolata & -0.29 \\
Serrapinnus sp. & -0.18 \\
Steindachnerina dobula & -0.15 \\
Steindachnerina guentheri & -0.15 \\
Synbranchus marmoratus & -0.30 \\
Trichomycterus sp. ${ }^{*}$ & 0.27 \\
Tyttocharax cf madeirae & -0.20 \\
\hline
\end{tabular}

* Densidad de especies que muestran correlaciones positivas con la altura.

* Species density showing positive correlation with altitude.

APÉNDICE 8

Perfiles longitudinal-altitudinal trazados por los ríos principales de cada una de las dos cuencas

APPENDIX 8

Altitudinal profile of the river mainstem for both drainages
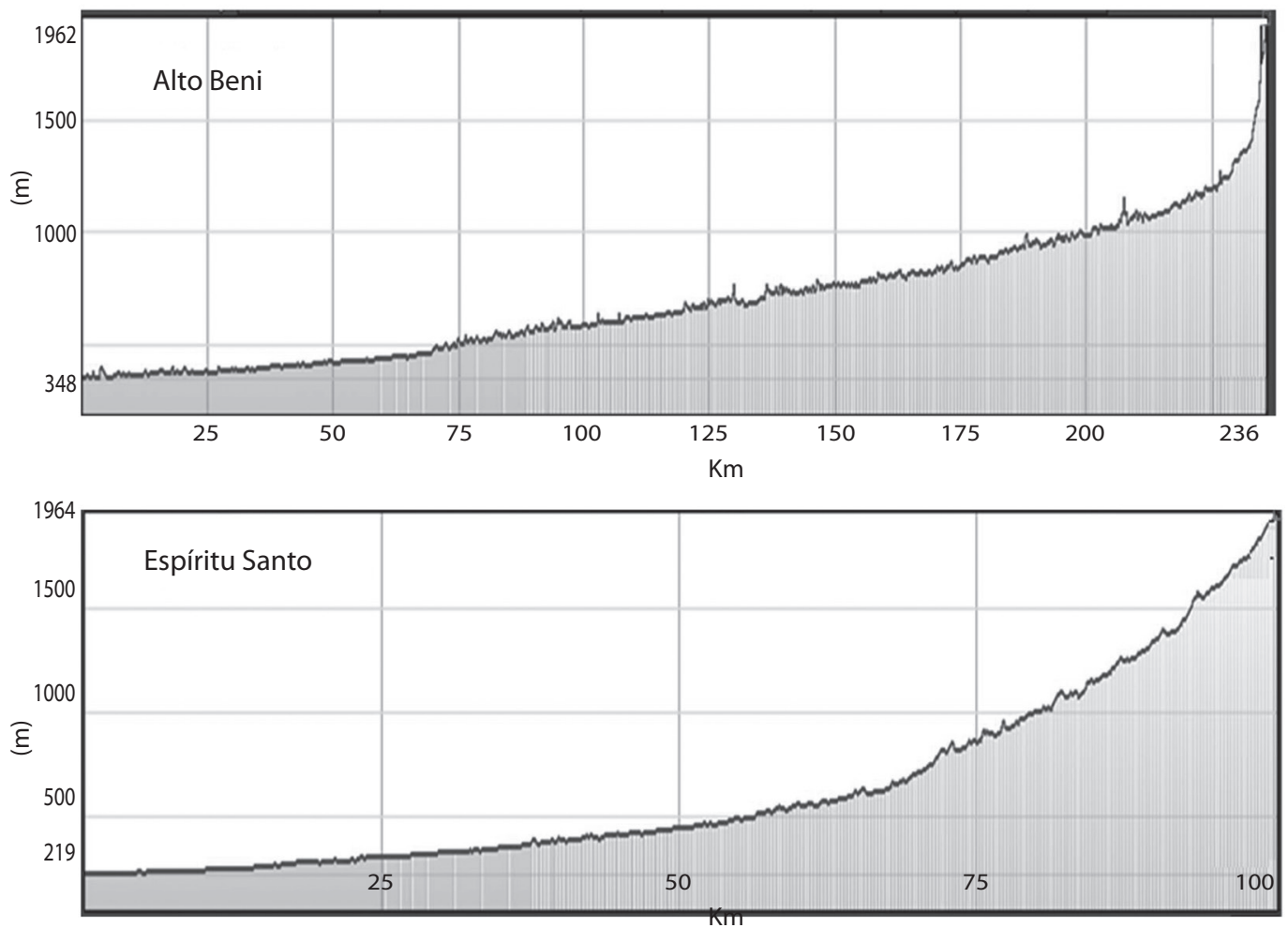